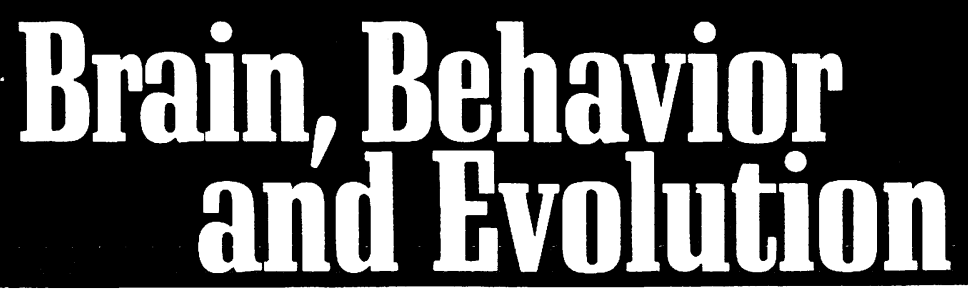

Vol. 36, No. 1, 1990

Thalamic Projections from a Midbrain Somatosensory Area in a Reptile, Caiman crocodilus

Pritz, M.B.; Stritzel, M.E. (Orange, Calif.)

Phylogeny of Putative Cholinergic Visual Pathways through the Pretectum to the Hypothalamus in Teleost Fish



Retinofugal Projections in a Marsupial, Tarsipes rostratus (Honey Possum)

Harman, A.M.; Coleman, L.-A.; Beazley, L.D. (Nedlands) . . . . . . . . . . . . . . . . . . 30

Afferent Connections of the Striatum and the Nucleus accumbens in the Lizard Gekko gecko

Gonzalez, A.; Russchen, F.T.; Lohman, A.H.M. (Madrid/Amsterdam)

Distribution of Phenylethanolamine-N-Methyltransferase-Immunoreactive Perikarya and Fibers in the Brain of the Lizard Gekko gecko

Smeets, W.J.A.J.; Jonker, A.J. (A.msterdam) 


\title{
Phylogeny of Putative Cholinergic Visual Pathways through the Pretectum to the Hypothalamus in Teleost Fish
}

\author{
Mario F. Wullimann, Dietrich L. Meyer \\ Department of Neuroanatomy, School of Medicine, University of Göttingen, Göttingen, FRG
}

Key Words. Acetylcholinesterase $\cdot$ Evolution $\cdot$ Hypothalamus $\cdot$ Phylogeny $\cdot$ Pretectum $\cdot$ Teleost $\cdot$ Visual system

\begin{abstract}
Three patterns of pretectal organization can be discerned morphologically in teleosts. The taxonomic distribution of these pretectal patterns suggests that the intermediately complex pattern (seen in most teleost groups) has given rise to both the elaborate pattern (seen in percomorphs) and the simple pattern (seen in cyprinids). Two pretectal patterns (intermediately complex and elaborate) form part of similar, homologous visual pathways to the hypothalamus; the third pattern is involved in a nonhomologous pathway to the hypothalamus. Acetylcholinesterase (AChE) histochemistry was used in the present study in order to characterize these pretectal patterns further. It is demonstrated that AChE is a highly selective and reliable interspecific marker for all divisions of the superficial pretectum, the nucleus corticalis, the posterior pretectal nucleus (or nucleus glomerulosus) and portions of the inferior lobe. Therefore, the histochemical data support the hypothesis of a homology between the three patterns of pretectal organization in teleosts. Furthermore, the present data provide a basis for more specific investigations regarding the involvement of acetylcholine as a neurotransmitter within the visual pathways to the hypothalamus in teleosts.
\end{abstract}

\section{Introduction}

At least three major patterns of pretectal organization exist within teleost fish (fig. 1). Apparently, the most elaborate pattern occurs in percomorph teleosts (fig. 1b), where some pretectal nuclei form relay centers within visual pathways to the hypothalamus. Morphological and hodological work in percomorphs has shown that a complex array of nuclei and fiber tracts constitutes visual pathways leading from retinal ganglion cells through several pretectal nuclei to the inferior lobe of the hypothalamus [Sakamoto and Ito, 1982; Murakami et al., 1986; Striedter and Northcutt, 1986, 1989; Wullimann and Northcutt, 1986; for a review, see Northcutt and Wullimann, 1988]. The nucleus glomerulosus is the most complex relay center within these pathways. This large and layered nucleus is bilobed, with a smaller rostral lobe extending to- ward the superficial pretectum and-a larger caudal lobe extending far back into the preglomerular cell masses.

There are two possible homologues of this elaborate percomorph pretectal pattern in other teleosts. One is found in some ostariophysines, e.g. cyprinids. It consists of fewer and less conspicuously organized nuclei (fig. 1c). A phyletic analysis has previously shown that many visual nuclei in the diencephalon of cyprinids are not evolutionarily primitive but were reduced secondarily during the ostariophysine evolution; i.e. they represent a derived condition [Northcutt and Wullimann, 1988]. This pattern of pretectal organization in cyprinids will be referred to here as the simple pattern.

A second possible homologue of the elaborate percomorph pretectal pattern is seen in most other teleostean groups, such as osteoglossomorphs, esocids and 
salmonids (fig. 1a, d); it is of intermediate complexity compared to the other two patterns (fig. 1a). This intermediately complex pattern is characterized by a large posterior pretectal nucleus which occupies a position similar to that of the nucleus glomerulosus in the percomorph pattern. However, its histology is distinctly different since its neural components are not organized in layers and since it does not extend caudally into the preglomerular cell masses.

Although there is considerable morphological variation among the three patterns, the general topology suggests the hypothesis that they represent different morphotypes of a homologous set of nuclei. A related, but different, topic is the phylogenetic relationship between the patterns. Once a hypothesis of homology between the patterns is corroborated by structural similarities, the systematic distribution of the patterns should be considered. The posterior pretectal nucleus is not as large in elopomorphs and some clupeomorphs as it is in most osteoglossomorphs, esocids or salmonids [M.F. Wullimann, unpubl. observ.]. However, the former two teleostean groups also show a variation of the intermediately complex pattern of pretectal organization. A survey of the pretectal morphology within the major teleostean groups [Northcutt and Wullimann, 1988; Butler et al., 1989; A.B. Butler, M.F. Wullimann and R.G. Northcutt, unpubl. observ.] strongly indicates that both the simple and elaborate patterns are derived from the intermediately complex one, since the latter is found in all outgroups of the percomorphs and ostariophysines (fig. 1d).

Two approaches to test the hypothesis of homology between the pretectal patterns seem obvious: to look for similarities in connections and for similarities in histochemistry. Recently, we have investigated the visual circuitry to the hypothalamus in an osteoglossomorph, Osteoglossum bicirrhosum, which shows the intermediately complex pattern (fig. la). We have demonstrated that the connections of the posterior pretectal nucleus are identical to those of the nucleus glomerulosus and intermediate superficial pretectal nucleus [Wullimann and Northcutt, 1989]. This detailed similarity of connections supports the hypothesis that the posterior pretectal nucleus is homologous to the nucleus glomerulosus and intermedi-

Abbreviations used in figures $1-12$

\begin{tabular}{|c|c|c|c|}
\hline A & Nucleus anterior thalami & MO & Medulla oblongata \\
\hline AOD & Nucleus accessorius opticus dorsalis & NF & Nucleus of the medial longitudinal fasciculus \\
\hline AOV & Nucleus accessorius opticus ventralis & OT & Optic tract \\
\hline AP & Nucleus pretectalis accessorius & $P$ & Nucleus pretectalis superficialis, pars parvocellularis \\
\hline $\mathrm{C}$ & Nucleus corticalis & $\mathrm{PC}$ & Nucleus paracommissuralis \\
\hline $\mathrm{Ce}$ & Corpus cerebelli & PG & Nucleus preglomerulosus \\
\hline $\mathrm{CM}$ & Corpus mammillare & PLI & Nucleus periventricularis lobi inferiores \\
\hline $\mathrm{CS}$ & Commissura supraoptica & PP & Nucleus preopticus parvocellularis \\
\hline DLI & Nucleus diffusus lobi inferiores & PPd & Nucleus pretectalis periventricularis, pars dorsalis \\
\hline DOT & Dorsomedial optic tract & $\mathrm{PPv}$ & Nucleus pretectalis periventricularis, pars ventralis \\
\hline $\mathrm{E}$ & Eminentia ganularis & $\mathrm{SC}$ & Nucleus suprachiasmaticus \\
\hline FLL & Fasciculus longitudinalis lateralis & $\mathrm{T}$ & Tectum opticum \\
\hline FR & Fasciculus retroflexus & TA & Nucleus tuberis anterior \\
\hline GT & Nucleus gustatorius tertius & TMC & Tractus mesencephalocerebellaris \\
\hline $\mathrm{Ha}$ & Habenula & TP & Nucleus tuberis posterior \\
\hline $\mathrm{Hv}$ & Ventral periventricular hypothalamus & TS & Torus semicircularis \\
\hline Hy & Hypophysis & $\mathrm{V}$ & Vagal lobe \\
\hline I & Nucleus pretectalis superficialis, pars intermedius & VL & Nucleus ventrolateralis thalami \\
\hline LH & Nucleus lateralis hypothalami & VM & Nucleus ventromedialis thalami \\
\hline LI & Lobus inferior & VOT & Ventrolateral optic tact \\
\hline M & Nucleus pretectalis superficialis, pars magnocellularis & & \\
\hline
\end{tabular}


a Primitive teleostean condition

b Derived percomorph condition

C Derived cyprinid condition
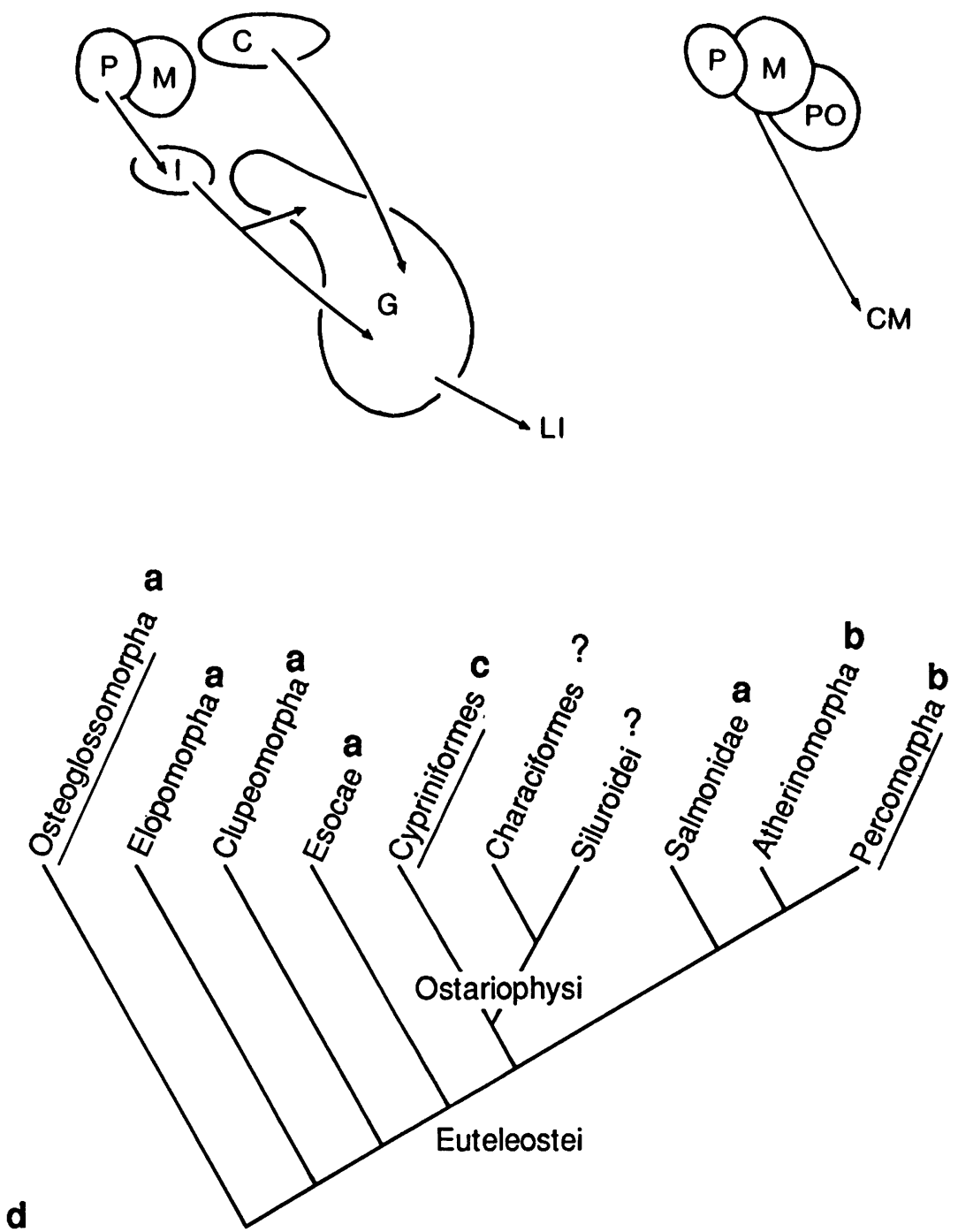

Fig. 1. a-c Schematic illustrations of the three patterns of pretectal organization in teleosts. a Intermediately complex pattern with arrows indicating connections according to Wullimann and Northcutt [1989]. b Elaborate pattern with arrows indicating connections as reviewed by Northcutt and Wullimann [1988]. c Simple pattern with arrow indicating a connection according to Northcutt and Braford [1984]. Based on its distribution (see d), pattern $\mathbf{a}$ is interpreted as the primitive one for teleosts, and patterns $\mathbf{b}$ and $\mathbf{c}$ are interpreted as derived ones. d Cladogram reflecting the phylogenetic relationships between teleost fish according to Lauder and Liem [1983]. The distribution of the patterns of pretectal organization (a-c in this figure) is indicated. The underlined taxa include the three species investigated in the present study. 

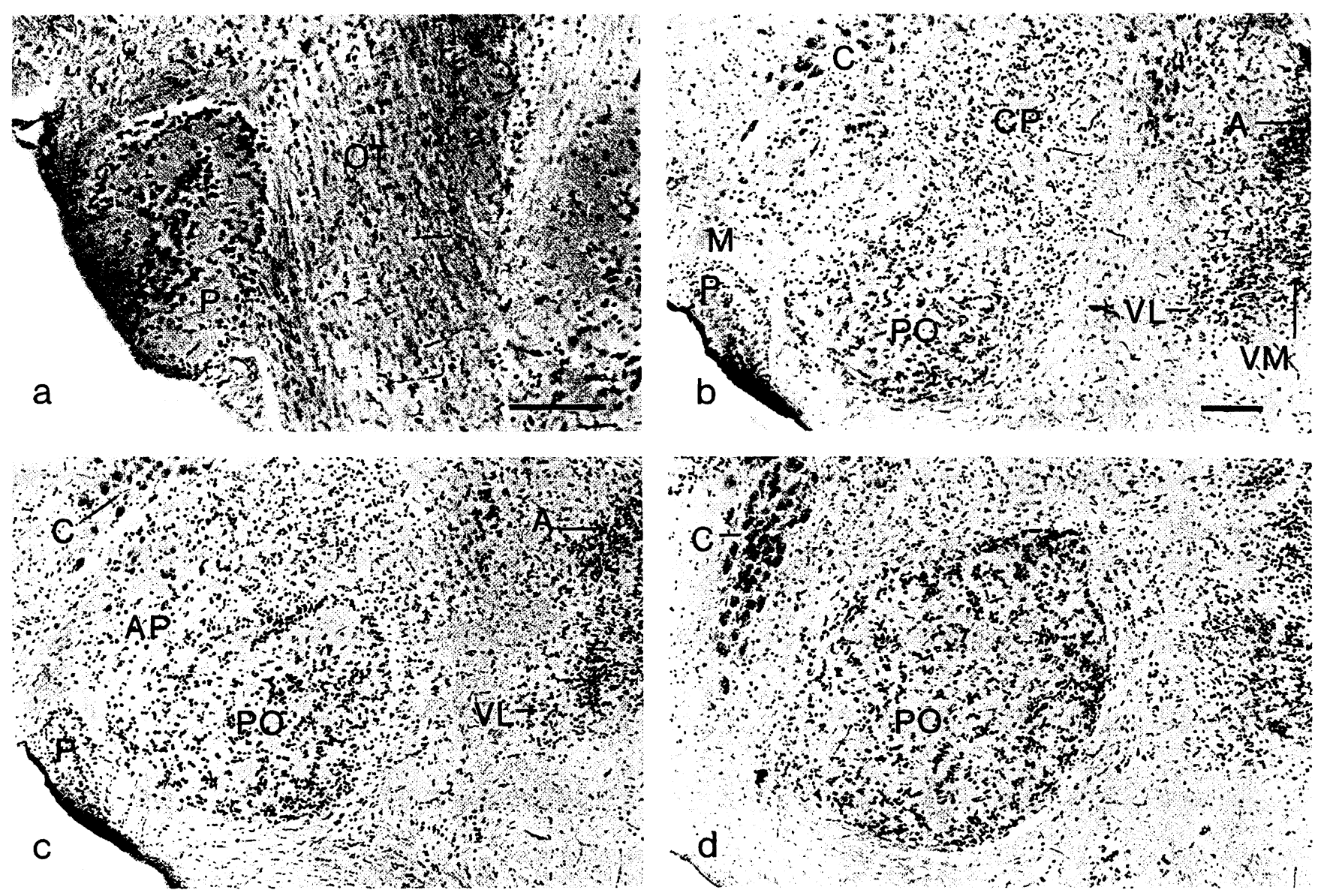

Fig. 2. Photomicrographs of Bodian-stained cross-sections showing the normal anatomy of the pretectum in Osteoglossum from rostral (a) to caudal (d). a, b Bars $=0.1 \mathrm{~mm}$. c, d Same magnification as b. Lateral is to the left and dorsal to the top in figures 2-4 and 6-11.

superficial pretectal nucleus (misidentified as central pretectal nucleus in Wullimann [1985]).

The present study provides histochemical information supporting the hypothesis of homology between the three patterns of pretectal organization. Ekström [1987] has shown that the neuropil of the parvocellular superficial pretectal nucleus and the neurons of the magnocellular superficial pretectal nucleus are strongly immunoreactive to choline acetyltransferase (ChAT) in Phoxinus phoxinus. This cyprinid species exhibits the simple pattern of pretectal organization. Thus, it is of interest to determine which portions of the pretectum in the intermediately complex and elaborate patterns contain acetylcholinesterase (AChE). Therefore, we chose a cyprinid, Carassius auratus, an osteoglossomorph, O. bicirrhosum, and a perco- morph, Hemichromis lifalili, as representatives of the simple, intermediately complex and elaborate patterns, respectively, and investigated the diencephalon and mesencephalon in these species with regard to the presence or absence of $\mathrm{AChE}$.

The neuroanatomical nomenclature follows that of Braford and Northcutt [1983] as modified by Northcutt and Wullimann [1988], except where noted. The taxonomic nomenclature is that of Lauder and Liem [1983]. A preliminary report on this topic has been published previously [Wullimann and Meyer, 1989].

\section{Materials and Methods}

Brain tissue was prepared either for histochemistry or for normal anatomical analysis. All animal research described in this 

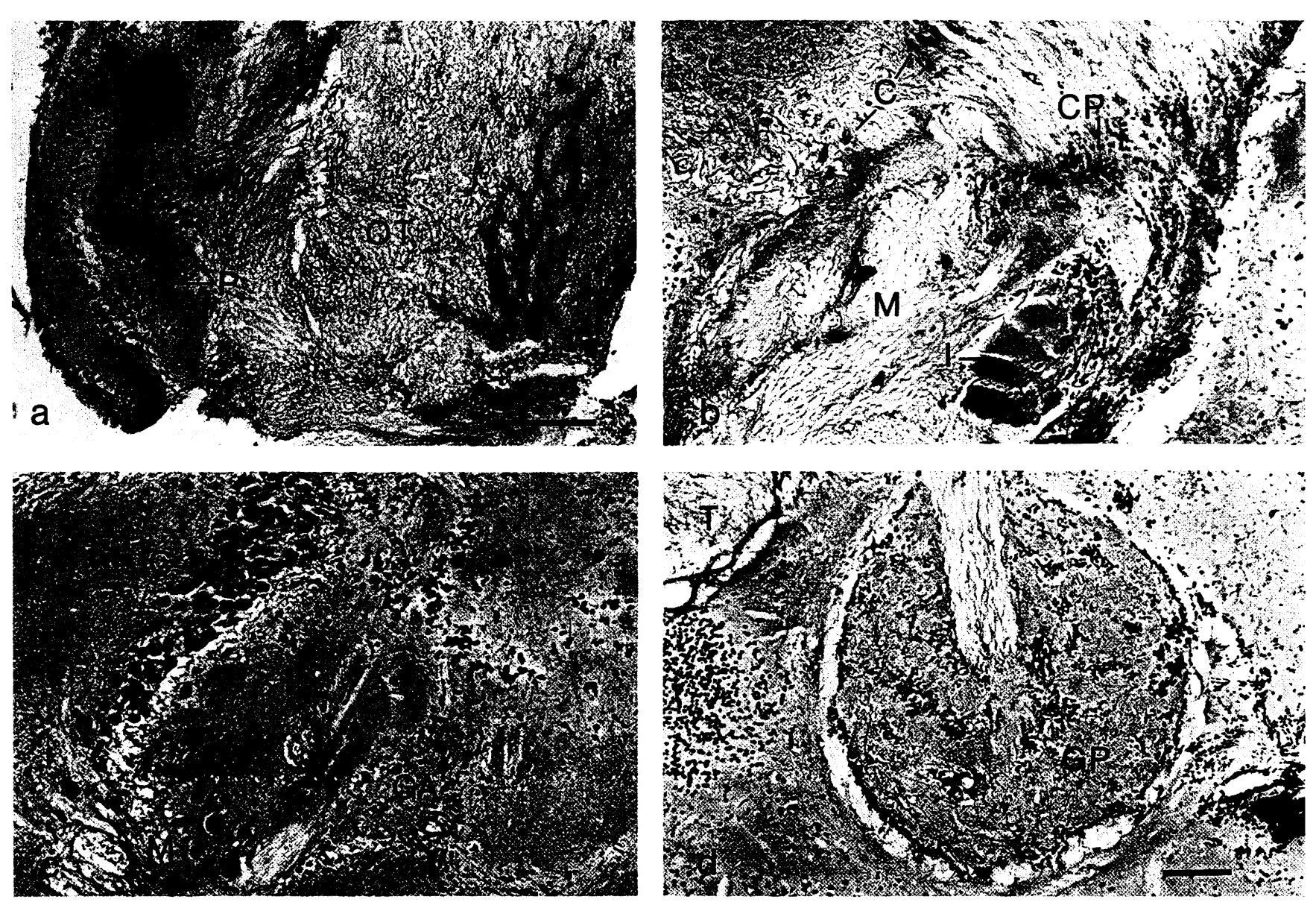

Fig. 3. Photomicrographs of Bodian-stained cross-sections showing the normal anatomy of the pretectum in Hemichromis from rostral (a) to caudal (d). a, d Bars $=0.1 \mathrm{~mm}$. b, c Same magnification as a.

study has been approved by the 'Regierungspräsident' of the State of Lower Saxony, FRG.

\section{Histochemistry}

Three specimens each of O. bicirrhosum, H. lifalili and C. auratus were anesthetized in a diluted solution of MS222 (Sigma, München, FRG) and perfused transcardially with cold teleostean Ringer's solution containing $1 \%$ heparin, followed by cold $10 \%$ formalin (Sigma) in $0.05 \mathrm{M}$ phosphate buffer. The brain, liver and, only in Osteoglossum, the gut were dissected out, postfixed for 3-8 $\mathrm{h}$ in the same fixative supplemented with $30 \%$ sucrose, and transferred to phosphate buffer with $30 \%$ sucrose overnight. The brains, livers and guts were then sectioned transversely $(30 \mu \mathrm{m})$ in a cryostat. The sections were reacted on the slides to detect the presence of AChE after the protocol of Lynch and Killackey [1974].

As controls, promethazine (Sigma), an inhibitor of unspecific cholinesterases (butyrylcholinesterase; $\mathrm{BuChE}$ ), was added to the incubation solution in parallel experiments. There was no effect on the histochemical staining pattern of the brain tissue. Furthermore, when acetylthiocholine iodide was replaced by S-butyrylthiocholine iodide (both from Sigma) as a substrate for cholinesterases, no staining was observed in any of the brain tissues at all.

The liver and gut, which have been claimed to contain BuChE [Koelle, 1950, 1951, 1963], were also investigated for the presence of unspecific cholinesterases to provide a further check for the apparent absence of BuChE in the brain tissue. Only the intestinal smooth muscles of Osteoglossum showed evidence for the presence of BuChE. The use of S-butyrylthiocholine iodide produced heavy staining of these muscles, whereas the use of acetylthiocholine iodide yielded only weak staining. A similar staining pattern was achieved when promethazine was used in conjunction with S-butyrylthiocholine iodide. By omitting both acetylthiocholine iodide and S-butyrylthiocholine iodide, no smooth muscle staining was observed at all. These control experiments indicate that the smooth musculature of the gut in Osteoglossum contains some AChE as well as a high amount of BuChE. Apparently, BuChE can be visualized with this method. However, there was no staining at all in the brain tissue in the three species investigated when S-butyrylthio- 

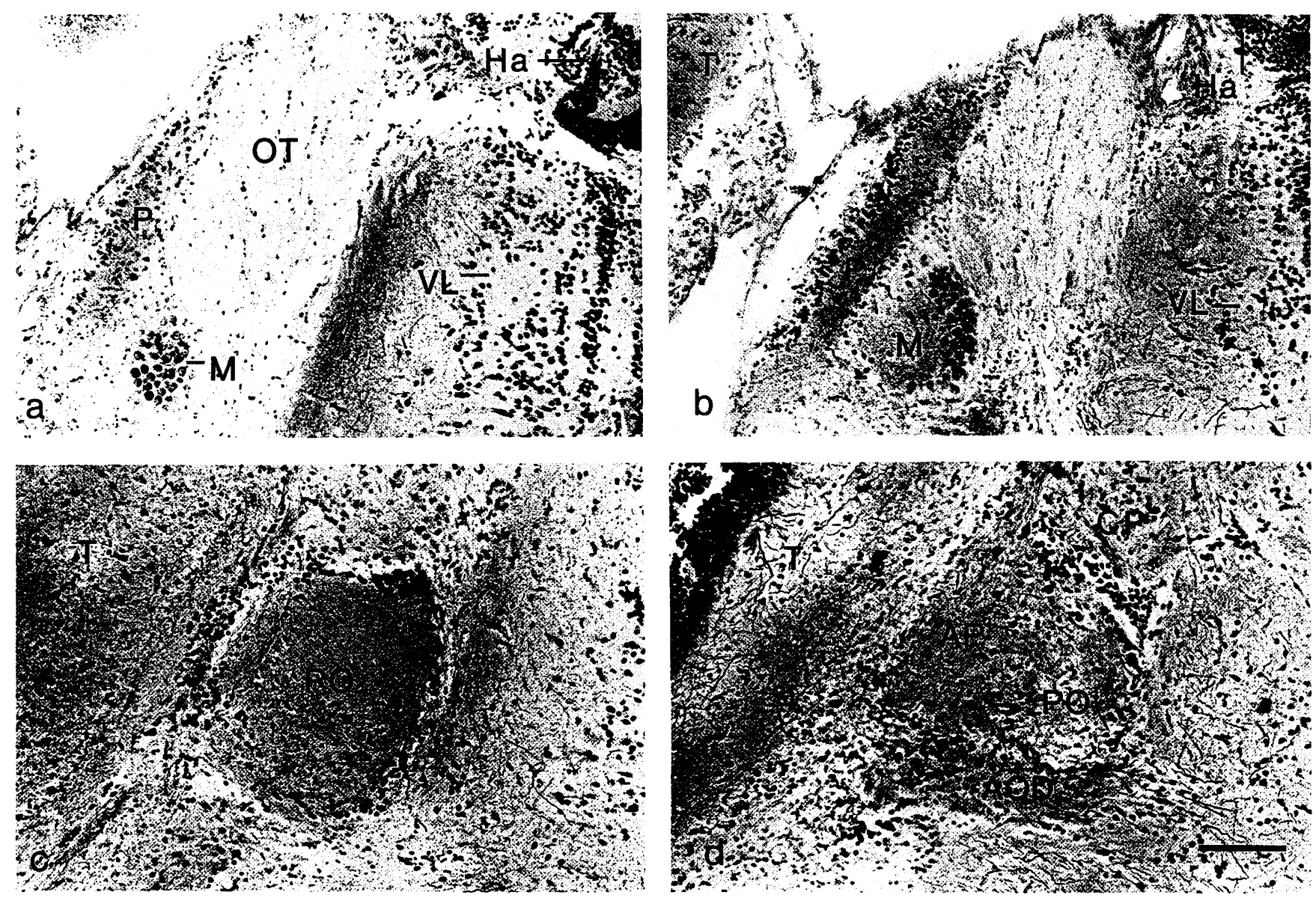

Fig. 4. Photomicrographs of Bodian-stained cross-sections showing the normal anatomy of the pretectum in Carassius from rostral (a) to caudal (d). Bar in d equals $0.1 \mathrm{~mm}$ and applies to a-d.

choline iodide was used, in contrast to the heavy staining when acetylthiocholine iodide was used. Therefore, the controls also show that all histochemical staining observed in the brain was due to the presence of AChE.

\section{Normal Anatomy}

In addition to the specimens examined for AChE histochemistry, one specimen each of the three species was perfused in the same way as described above, except that AFA was used as a fixative ( $90 \mathrm{ml} \mathrm{80 \%} \mathrm{ethanol,} 5 \mathrm{ml}$ formalin, $5 \mathrm{ml}$ glacial acetic acid). The brains were postfixed in AFA for at least 1 month before they were embedded in paraffin, cut transversely at $15 \mu \mathrm{m}$ and silver-stained with Protargol (Roques chimie, St. Ouen, France) according to the Bodian method [Romeis, 1989].

\section{Results}

The normal anatomy of the pretectum is described in detail in the three species investigated, followed by a description of the AChE distribution in the diencephalon and mesencephalon of these teleosts.

\section{Normal Anatomy}

The normal anatomy of the pretectum in the three species is illustrated in figures $2-4$.

Osteoglossum. This osteoglossomorph species shows the intermediately complex pattern of pretectal organization (fig. 2). The parvocellular superficial pretectal nucleus emerges furthest rostrally in the diencephalon and is embedded in the optic tract. It consists of small cells that rim a pleated band of central neuropil (fig. 2a). Caudally and dorsomedially, a small magnocellular superficial pretectal nucleus emerges. The posterior pretectal nucleus lies ventral to both nuclei; it is histologically distinct with smaller, regularly clustered neurons. This nucleus is the largest and most caudally extending pretectal nuclear group 

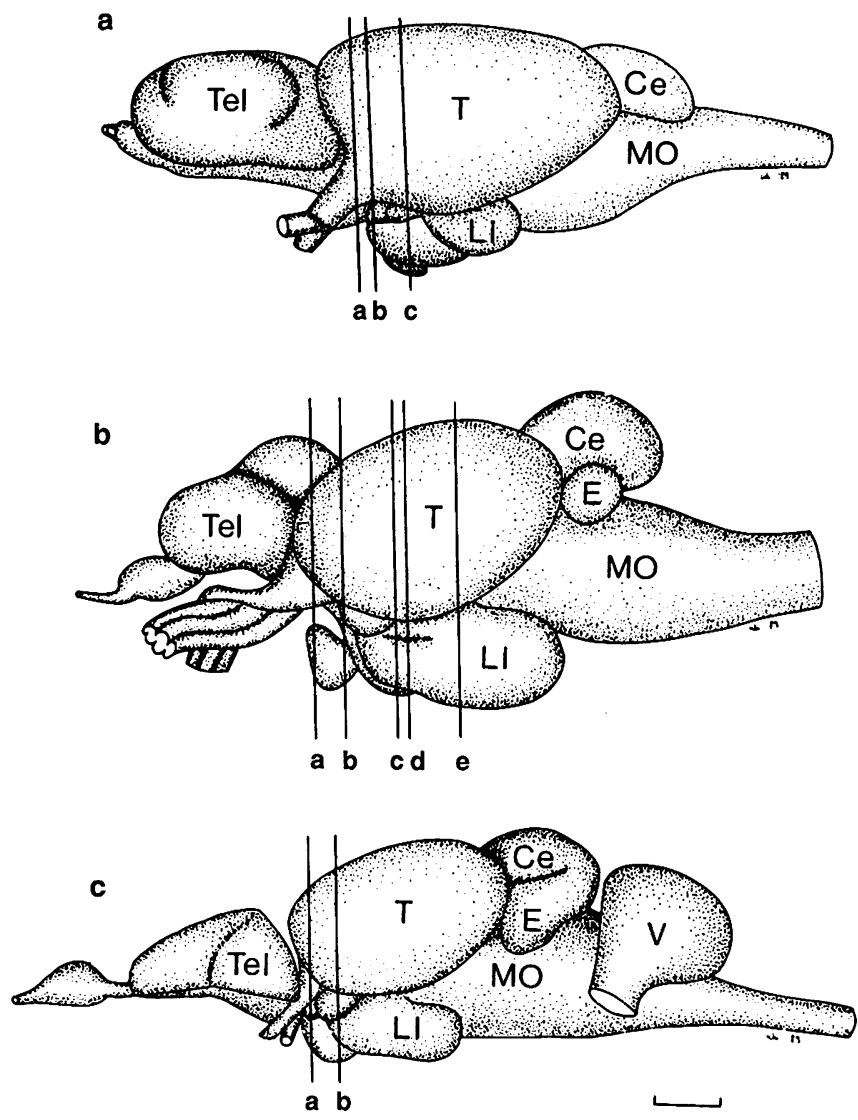

Fig. 5. Lateral views of the brains of Osteoglossum (a), Hemichromis (b) and Carassius (c) indicating the positions of the crosssections shown in figures $6-8$, respectively. Most cranial nerves have been omitted. Bar $=1 \mathrm{~mm}$.

in Osteoglossum. The nucleus corticalis is located dorsal to the magnocellular superficial pretectal nucleus. The huge neurons of this nucleus lie at the border to the optic tectum (fig. 2b-d; 6b, c). At more caudal levels, the magnocellular superficial pretectal nucleus is replaced by a smaller-celled nucleus, the accessory pretectal nucleus, which lies sandwiched between the nucleus corticalis and the posterior pretectal nucleus (fig. 2b-d). The more medially located central pretectal nucleus is also situated between these two nuclei (fig. 2b). The dorsal and ventral parts of the periventricular pretectum are found even more medially and caudally, and are characteristically located around the fasciculus retroflexus.

Hemichromis. This percomorph species displays the elaborate pattern of pretectal organization (fig. 3). Most rostrally, a complexly pleated parvocellular su- perficial pretectal nucleus emerges within the optic tract. Its neurons are located at the rim of a neuropil band in a characteristic fashion (fig. 3a). More caudally and dorsomedially, it is replaced by the largecelled magnocellular superficial pretectal nucleus and ventromedially by the intermediate superficial pretectal nucleus (fig. 3b). The latter is replaced caudally by the anterior part of the glomerular nucleus (fig. 3c). This nucleus is easily identifiable by its specialized neuropil which is organized in spherical glomeruli (fig. 10c). These glomeruli continue to be present more caudally within the posterior part of the nucleus glomerulosus, which extends caudally into the preglomerular cell masses and approaches the inferior lobe of the hypothalamus. The nucleus glomerulosus displays small and large cells. The small cells are found throughout the nucleus, whereas the large cells are mostly found in the periphery (fig. 3d). This histological organization is a variation of the incompletely laminated type of the nucleus glomerulosus (= nucleus rotundus of Ito and Kishida [1975]). Dorsomedial to the magnocellular superficial pretectal nucleus, a smaller-celled nucleus, the accessory pretectal nucleus, can be observed; it is located between the anterior part of the nucleus glomerulosus and the largecelled nucleus corticalis (fig. 3c). Even more medially the central pretectum (fig. 3b) and the periventricular pretectum are seen. The latter surrounds the fasciclus retroflexus.

Carassius. This cyprinid species shows the simple pattern of pretectal organization (fig. 4). Again, the parvocellular superficial pretectal nucleus is located most rostrally. Although not pleated, it consists of the characteristic neuropil band which is rimmed by small cells. Medial to this nucleus lies the large-celled magnocellular superficial pretectal nucleus (fig. 4a, b). More caudally it is replaced by the dorsomedially extending accessory pretectal nucleus and the ventrally located small posterior pretectal nucleus (fig. 4c, d). The latter nucleus disappears at a much more rostral level in Carassius than in Osteoglossum. The central pretectum lies medial to the accessory superficial and posterior pretectal nuclei (fig. 4d). Again, the periventricular pretectum is located around the fasciculus retroflexus. The nucleus corticalis is absent.

\section{AChE Activity}

The levels of the maps of AChE activity (fig. 6-8) described in the following section are depicted in a di- 


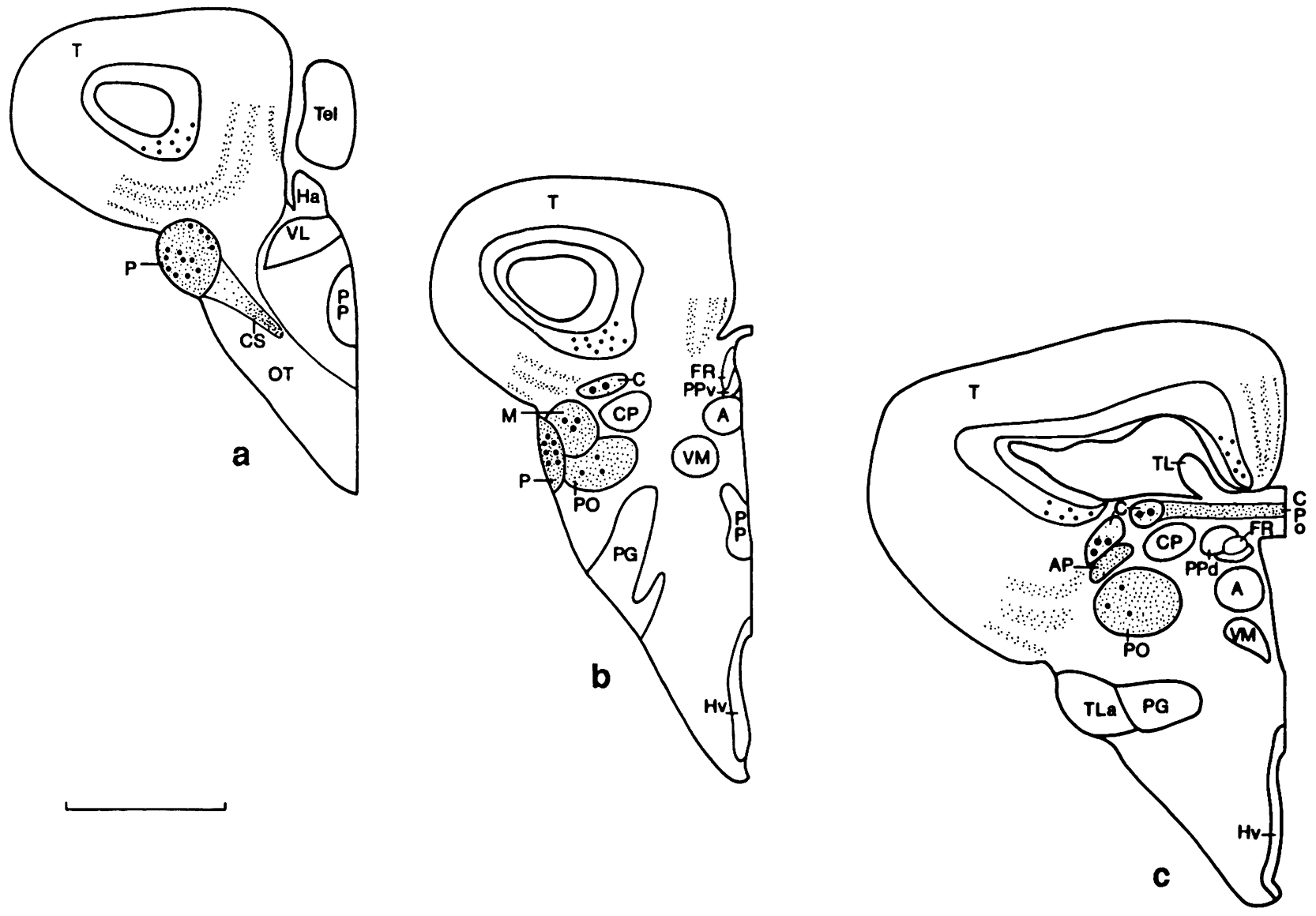

Fig. 6. Maps of AChE activity in the diencephalon and mesencephalon of Osteoglossum. Bar $=1 \mathrm{~mm}$.

agram of lateral views of the brains of Osteoglossum, Hemichromis and Carassius shown in figure 5.

Osteoglossum. In the pretectum, we found AChE reactivity in the neuropil and cell bodies of the parvocellular (fig. 6a; 9a) and magnocellular superficial pretectal nuclei, in the nucleus corticalis and in the posterior pretectal nucleus (fig. 6b; 9b-d), whereas only the neuropil of the accessory pretectal nucleus was labeled (fig. 6c; 9c, d). The AChE-containing neurons in the posterior pretectal nucleus were located in the lateral and rostral portions of the nucleus. Additional cell bodies were labeled in the periventricular gray zone of the optic tectum. Other AChE-containing fibers were seen in the posterior (fig. 6c) and supraoptic commissures (fig. 6a; 9b) and, further, in three distinct bands of the optic tectum, two within the superficial white and gray zones and one within the central zone. These AChE bands are continuous throughout the optic tectum in all three species inves- tigated. However, they are depicted only at the lateroventral and mediodorsal edges of the tectum in figures 6-8.

Hemichromis. In the pretectum, $\mathrm{AChE}$ reactivity was observed in the neuropil and cell bodies of the parvocellular (fig. 7a; 10a), magnocellular and intermediate superficial pretectum and in the nucleus corticalis (fig. $7 \mathrm{~b} ; 10 \mathrm{~b}$ ). In the accessory pretectal nucleus, such reactivity was restricted to the neuropil (fig. 7c; 10c). The anterior part of the nucleus glomerulosus, which extends toward the superficial pretectum (fig. 7c; 10c), continued to display strong label within specialized neuropil structures, the glomeruli, far caudally into the posterior part of the nucleus (fig. 7c-e; 10d). Additional AChE-containing fibers were observed in the supraoptic commissure (fig. 7a), the habenula (fig. 7c), the torus longitudinalis (fig. 7e), a restricted area within the ventrolateral optic tract (fig. $7 b-d$ ), the nucleus of the torus lateralis 

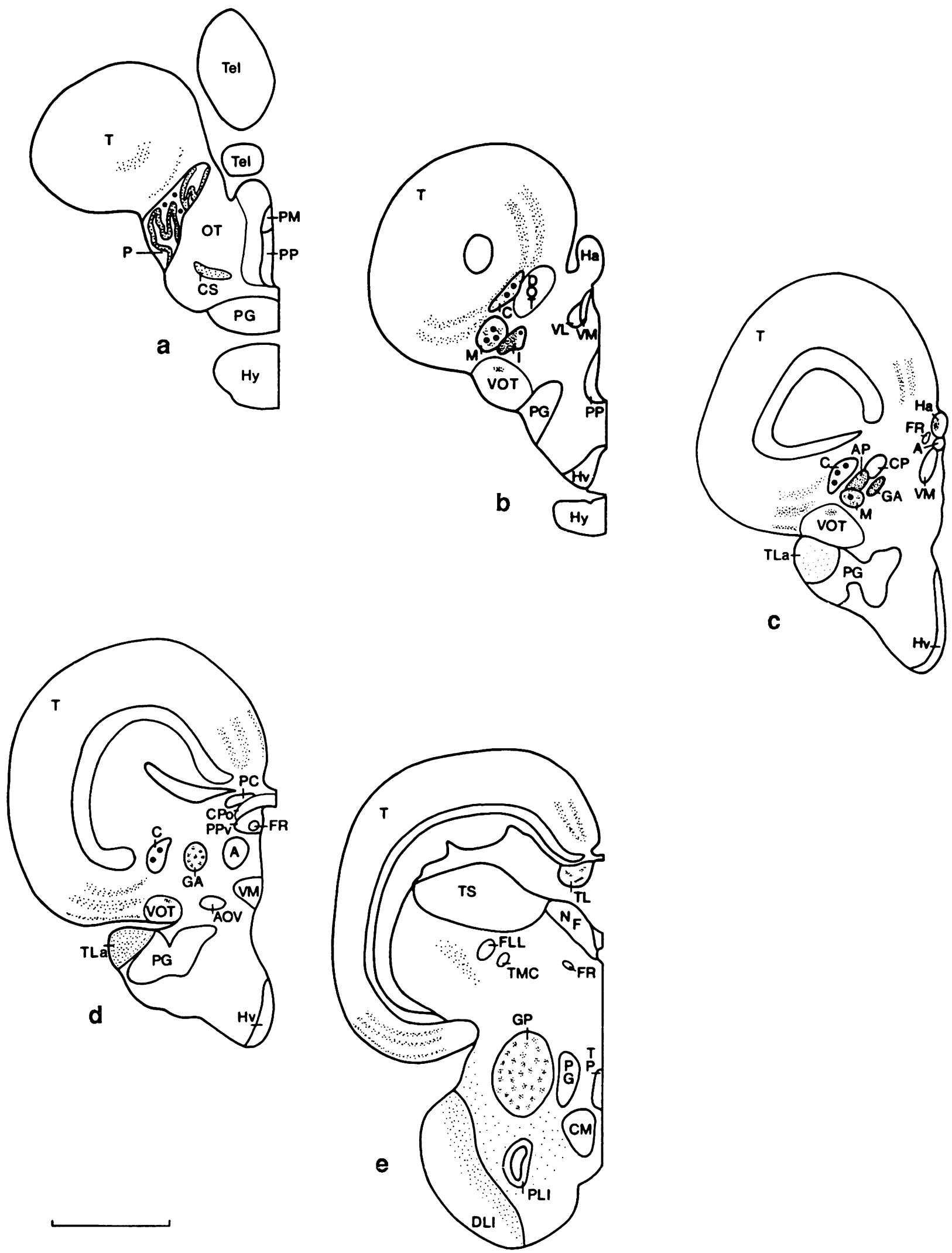

Fig. 7. Maps of AChE activity in the diencephalon and mesencephalon of Hemichromis. Bar $=1 \mathrm{~mm}$. 


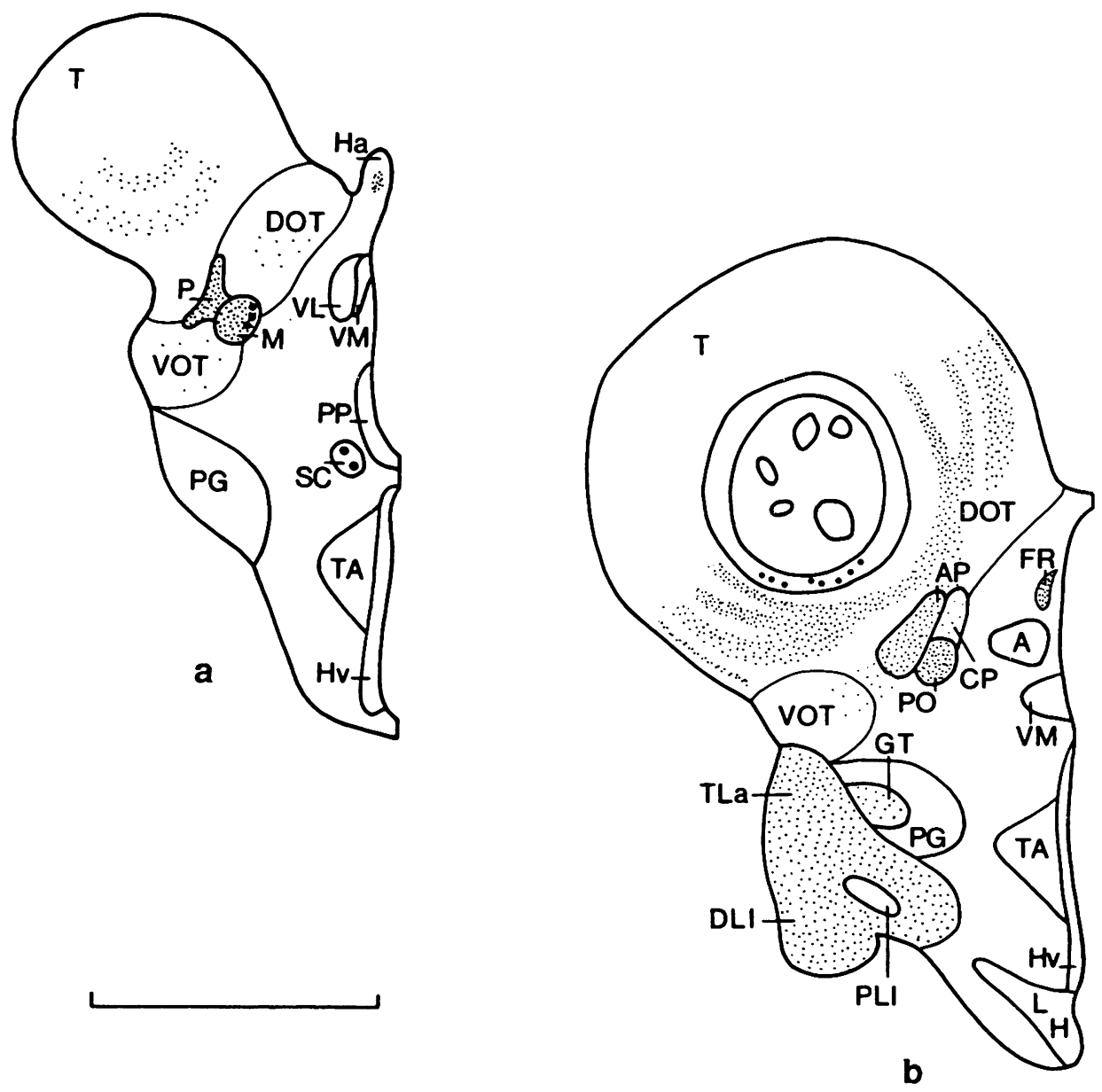

Fig. 8. Maps of AChE activity in the diencephalon and mesencephalon of Carassius. Bar $=1 \mathrm{~mm}$.

(fig. 7c, d), and, lightly, in the inferior lobe, particularly in the diffuse nucleus (fig. 7e). AChE-reactive fibers were also found in three distinct bands within the optic tectum, two within the superficial white and gray zones and one within the central zone.

Carassius. In the pretectum, AChE reactivity was seen in the neuropil of the parvocellular and magnocellular superficial pretectal nuclei (fig. $8 \mathrm{a} ; 11 \mathrm{a}, \mathrm{b}$ ), the accessory and posterior pretectal nuclei and, more lightly, in the central pretectum (fig. $8 b, 11 c$, d). The cell bodies of the magnocellular pretectum were also labeled (fig. $8 \mathrm{a} ; 11 \mathrm{a}, \mathrm{b}$ ). In Carassius, however, no continuation of AChE activity was observed as far caudally and ventrally as in Hemichromis or Osteoglossum. Additional cell bodies were labeled in the suprachiasmatic nucleus and in the periventricular gray zone of the optic tectum. Labeled fibers were observed in the habenula (fig. 8a), fasciculus retroflexus, nucleus of the lateral torus, diffuse nucleus of the inferior lobe and, more lightly, in the tertiary gustatory nucleus [see Wullimann, 1988] (fig. 8b). Furthermore, AChE-reactive fibers were seen in four bands within the optic tectum, two each in the superficial white and gray zones and in the central zone. Some labeled fibers were also seen in the ventrolateral and dorsomedial optic tracts.

In summary, these histochemical data reveal that, in teleosts, $\mathrm{AChE}$ is a highly specific and reliable interspecific marker for all divisions of the superficial pretectum, nucleus corticalis, posterior pretectal nucleus (or nucleus glomerulosus) and portions of the inferior lobe. 

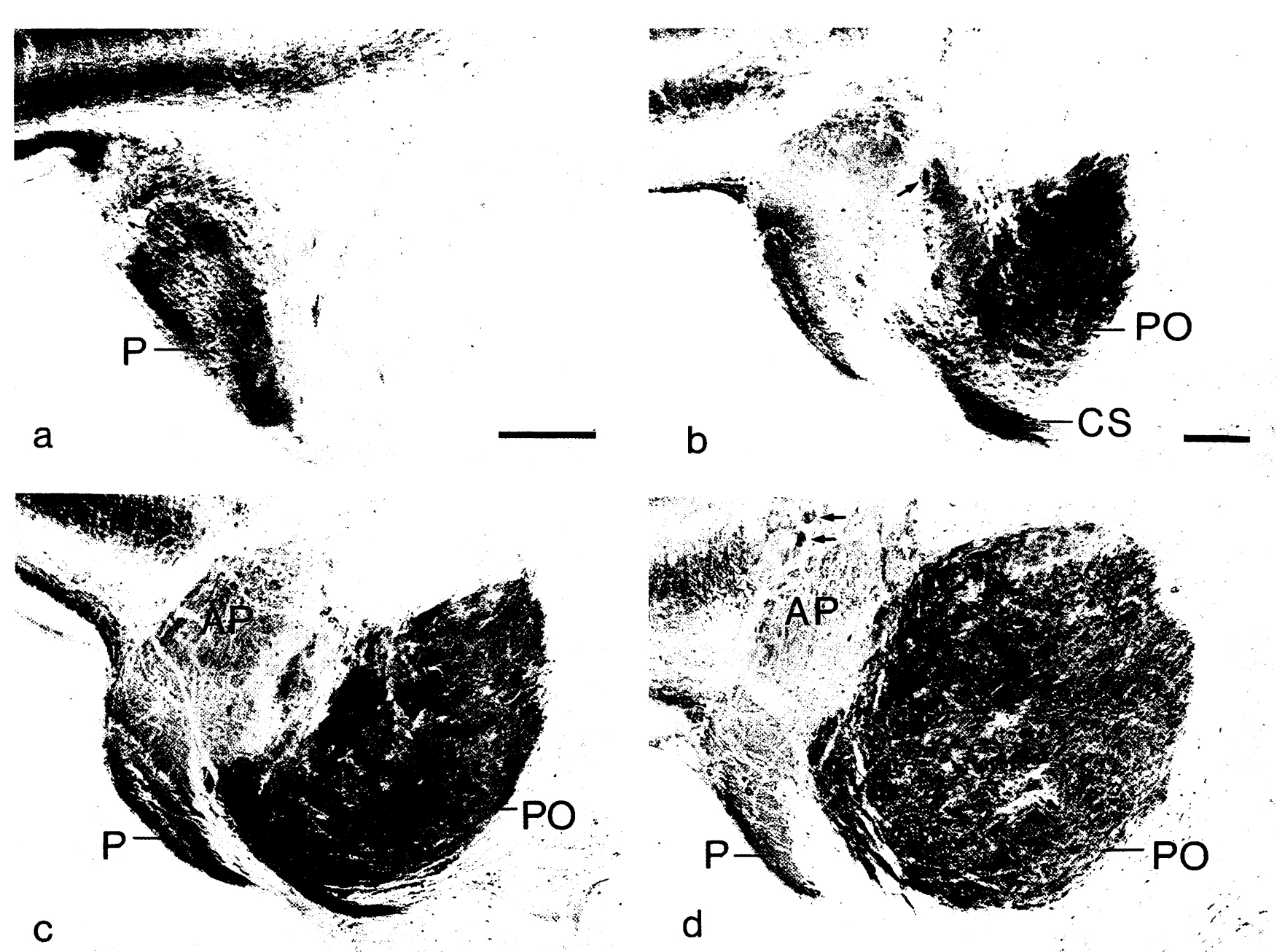

Fig. 9. Photomicrographs of cross-sections through the pretectum of Osteoglossum from rostral (a) to caudal (d) showing the AChE distribution. b Arrow points to a neuron in the magnocellular superficial pretectal nucleus. d Arrows mark nucleus corticalis neurons. a, b Bars $=0.1 \mathrm{~mm}$. c, d Same magnification as $\mathbf{b}$.

\section{Discussion}

The phylogenetic implications of the observed distribution of AChE activity in the diencephalon and mesencephalon of the three species investigated will be discussed first, followed by some functional considerations.

\section{Phylogenetic Considerations}

The neuronal circuitry in the three patterns of pretectal organization in teleosts (see fig. 1a-c) will be discussed and reviewed in detail in a companion paper reporting the neuroanatomical connections within the intermediately complex pattern [M.F. Wul- limann, D.L. Meyer and R.G. Northcutt, in preparation]. For the purpose of the present study, however, it is important to emphasize that the connections of the posterior pretectal nucleus in Osteoglossum are identical to those of the nucleus glomerulosus and intermediate superficial pretectal nucleus of percomorphs [Wullimann and Northcutt, 1989]. This observation supports the hypothesis that the anterior and posterior portions of the posterior pretectal nucleus are homologous to the intermediate superficial pretectal nucleus and nucleus glomerulosus of percomorph teleosts, respectively. Visual pathways to the hypothalamus homologous to those observed in the intermediately complex and elaborate patterns of pretectal 

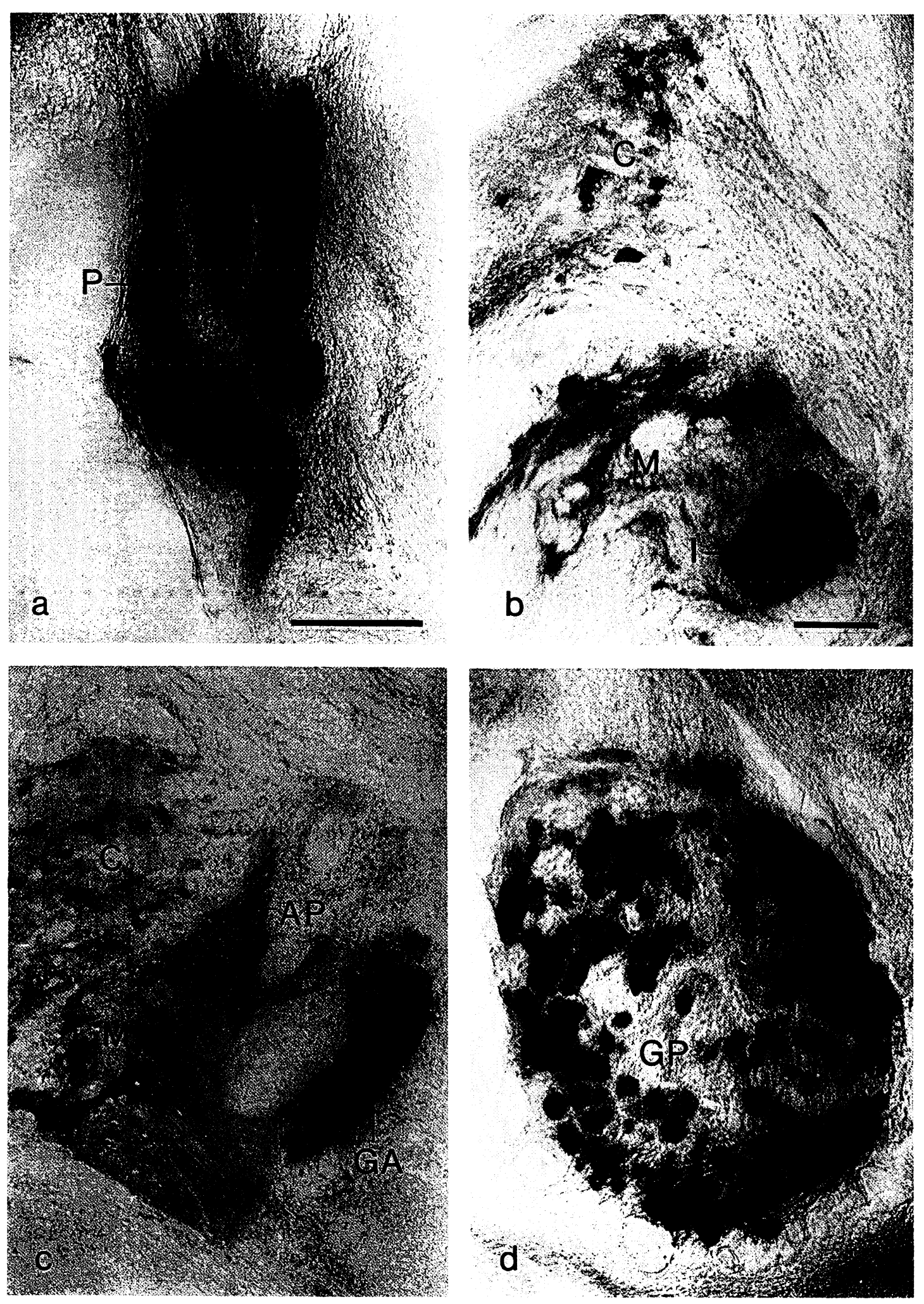

Fig. 10. Photomicrographs of cross-sections through the pretectum of Hemichromis from rostral (a) to caudal (d) showing the AChE distribution. a, b Bars $=0.1 \mathrm{~mm}$. c, $\mathbf{d}$ Same magnification as $\mathbf{b}$. 

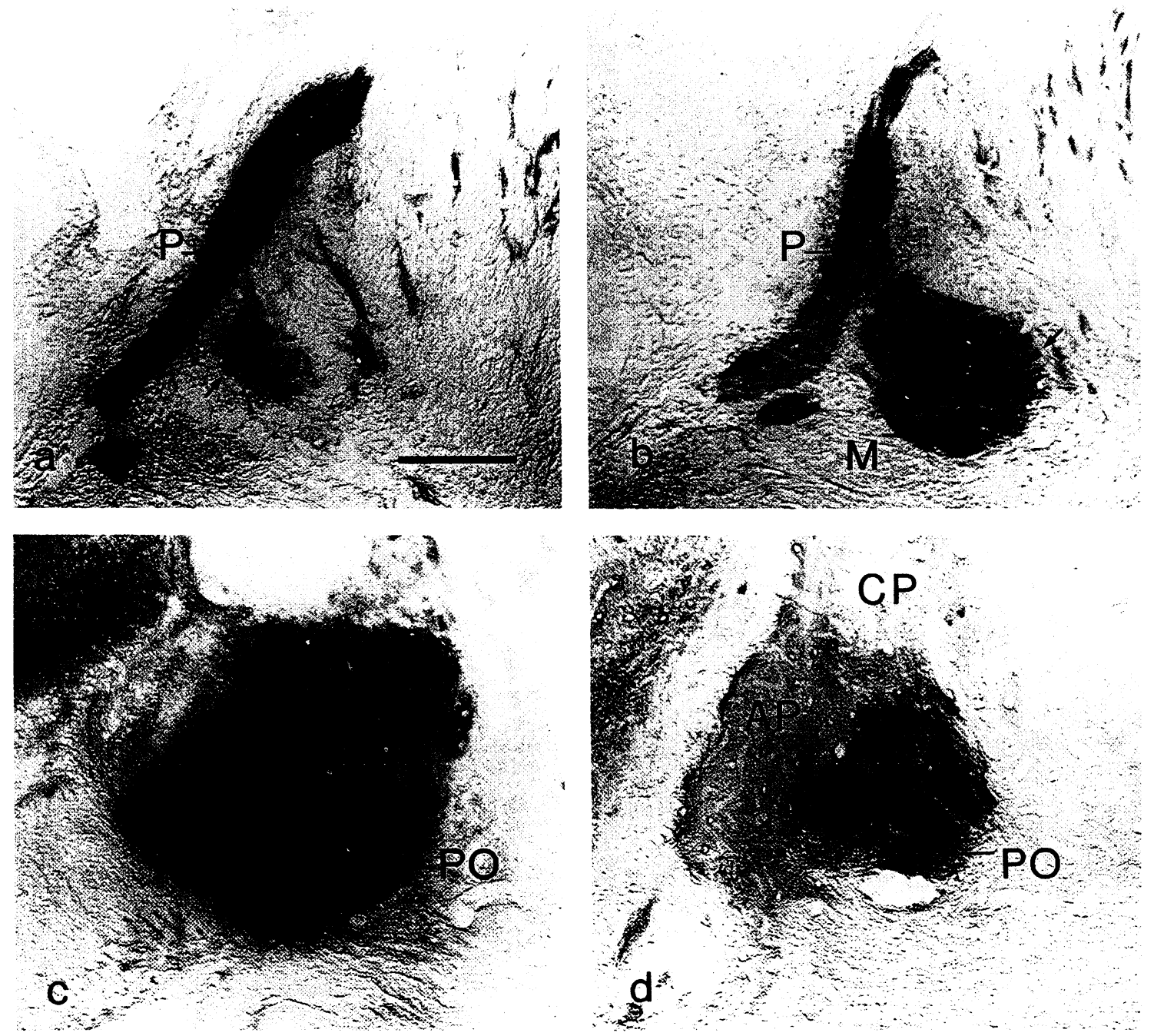

Fig. 11. Photomicrographs of cross-sections through the pretectum of Carassius from rostral (a) to caudal (d) demonstrating the AChE distribution. a, b Arrows point to neurons of the magnocellular superficial pretectal nucleus. Bar in a equals $0.1 \mathrm{~mm}$ and applies to a-d.

organization apparently do not exist in the simple pattern; however, a nonhomologous visual pathway (see fig. 1c) leading through the tectorecipient magnocellular superficial pretecal nucleus to the hypothalamus does exist in Carassius [Northcutt and Braford, 1984].

The present findings on AChE activity in the diencephalon and mesencephalon of three teleost species support the hypothesis of homology between the three patterns of pretectal organization to an even greater extent. The histochemical data demonstrate that AChE is a highly specific marker for all parts of the teleostean superficial pretectum, nucleus cortica- lis (when present), and posterior pretectal nucleus or nucleus glomerulosus (fig. 12). In conjunction with the known connectional information, the present data suggest several conclusions. (1) The parvocellular and magnocellular superficial pretectal nuclei and the nucleus corticalis (when present) are homologous between the three patterns. (2) The posterior pretectal nucleus in the intermediately complex and simple patterns is homologous to the nucleus glomerulosus and intermediate superficial pretectal nucleus in the elaborate pattern. In Osteoglossum, which displays the intermediately complex pattern, AChE-reactive cells were found in the rostrolateral portion of the 
posterior pretectal nucleus. In Hemichromis, which displays the elaborate pattern, such cells were seen in the intermediate superficial pretectum but not in the nucleus glomerulosus. Therefore, AChE histochemistry as well as the neuroanatomical connectivity support the hypothesis that the rostral and caudal portions of the posterior pretectal nucleus in the intermediately complex pattern are homologous to the intermediate superficial pretectum and nucleus glomerulosus, respectively, in the elaborate pattern. (3) There is an additional pretectal nucleus which we did not recognize previously, the accessory pretectal nucleus, which lies dorsomedial to the magnocellular superficial pretectal nucleus. It contains dense AChE activity in its neuropil in all three species and is therefore considered to be homologous between the three patterns.

In summary, AChE histochemistry of the three patterns of pretectal organization and the neuroanatomical connections in the intermediately complex and elaborate patterns reveal a detailed structural similarity. This is a prerequisite for a hypothesis of homology between them. The phylogenetic relationships between the patterns can therefore be considered by examining their systematic distribution (fig. 1d). The latter suggests first that the posterior pretectal nucleus is the ancestral condition for teleosts. It exhibits all connections of the nucleus glomerulosus and intermediate superficial pretectal nucleus of derived percomorph teleosts and, therefore, has probably given rise to both nuclei in percomorphs. The second suggestion is that, in Carassius, the posterior pretectal nucleus is reduced to a small nucleus located caudal to the magnocellular superficial pretectal nucleus. Crucial to this interpretation is whether or not the posterior pretectal nucleus of Carassius projects to the inferior lobes. In most teleosts it is almost impossible to expose surgically the inferior lobe in order to inject it with a conventional neuronal tracer. With the newly available DiI technique [Godement et al., 1987], however, this experiment can be performed in vitro.

\section{Functional Considerations}

Although the presence of immunoreactivity to ChAT, the acetylcholine-synthesizing enzyme, is a more reliable indicator for cholinergic pathways, AChE histochemistry remains valuable as a first approach to localize such pathways in the central nervous system [Butcher, 1983; Bradford, 1986]. The

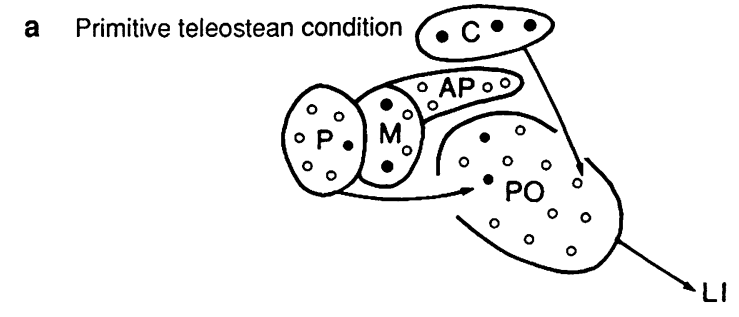

b Derived percomorph condition

C Derived cyprinid condition
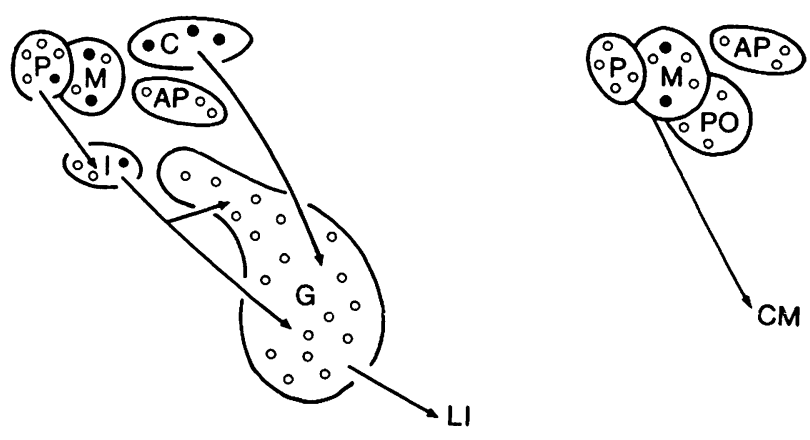

Fig. 12. Diagram summarizing the new information on $\mathrm{AChE}$ activity within the three patterns of pretectal organization $(\mathbf{a}-\mathbf{c})$. Unlike the maps shown in figures 6 and 7, AChE-reactive fibers in the nucleus corticalis are not indicated here (see Discussion). Filled circles represent cells; open circles represent neuropils.

present data reveal for the first time that some teleosts possess visual pathways to the hypothalamus that can selectively be characterized by AChE histochemistry. These data represent the basis for more specific hypotheses of the involvement of a cholinergic circuitry in the visual pathways to the hypothalamus in teleosts. These hypotheses can be tested further, e.g. by ChAT immunohistochemistry.

The AChE bands and AChE-containing neurons in the optic tectum observed in this study, however, have been reported and corroborated by ChAT immunohistochemistry in teleosts previously [Ekström, 1987; Zottoli et al., 1987]. In these and in the present studies, there is a topological correspondence between three retinal input layers $(\mathrm{t} 1, \mathrm{t} 2$ and $\mathrm{t} 3$ of Northcutt [1983]; stratum opticum, stratum fibrosum et griseum superficiale and stratum griseum centrale of von Bartheld and Meyer [1987]) and the discussed AChE bands. It has been argued that retinotectal terminals may be cholinoceptive [Zottoli et al., 1987]. Alternatively, it has been proposed that acetylcholine is used 
as neurotransmitter in the retinotectal system in teleosts [Contestabile et al., 1980; Oswald and Freeman, 1980] and amphibians [Oswald et al., 1979; Schmidt et al., 1989]. Whatever the case may be, the involvement of acetylcholine as a neurotransmitter of retinal ganglion cells seems to be restricted to teleosts and amphibians, and forms a major difference in the visual systems between these and other vertebrates [Oswald and Freeman, 1980; Wächtler, 1988] such as birds [Sorenson et al., 1989] or mammals [Bigl and Schober, 1977; Fibiger, 1982]. Similarly, the abundant presence of $\mathrm{AChE}$ within the visual pathways to the hypothalamus reported in this study may be a unique adaptation in teleosts.

AChE-reactive fibers in the nucleus corticalis lie in the direct continuation of the AChE-reactive band of the central zone of the optic tectum. They may, therefore, not be intrinsic to the nucleus corticalis.

More specific hodological and ChAT immunohistochemical analyses are needed in order to elucidate the cholinergic circuitry within the visual pathways to the hypothalamus in teleosts. However, one subcircuit in the intermediately complex and elaborate patterns should be considered a good candidate for a cholinergic projection: the axons of neurons of the nucleus corticalis in percomorphs project into the nucleus glomerulosus where they form the glomeruli [Sakamoto and Ito, 1982]. In Osteoglossum, which displays the intermediately complex pattern, nucleus corticalis neurons project into the posterior pretectal nucleus [Wullimann and Northcutt, 1989]. In this osteoglossomorph species, the nucleus corticalis neurons and the neuropil of the posterior pretectal nucleus contain a high amount of AChE. The same is true for neurons of the nucleus corticalis and the glomeruli of the nucleus glomerulosus in the percomorph Hemichromis. Therefore, both neuroanatomy and histochemistry suggest together that at least this portion of the visual pathways leading to the hypothalamus in teleosts may be cholinergic.

\section{Acknowledgements}

We would like to thank S. Kötting and K. Sebralla for technical assistance, I. Chao for raising some of the Hemichromis specimens used in this study, R. Dungan and S. Ruelke for making the photographic prints, and a reviewer for suggesting to treat 'pretectal patterns' separately from 'visual hypothalamic pathways'. This work was supported by the Swiss National Science Foundation (M.F.W.) and the German Science Foundation (D.L.M.).

\section{References}

Bartheld, C.S. von, and D.L. Meyer (1987) Comparative neurology of the optic tectum in ray-finned fishes: patterns of lamination formed by retinotectal projections. Brain Res., 420: 277-288.

Bigl, V., and W. Schober (1977) Cholinergic transmission in subcortical and cortical visual centers of rats: no evidence for the involvement of primary optic system. Exp. Brain Res., 27: 211 219.

Bradford, H.F. (1986) Chemical Neurobiology. Freeman, New York.

Braford, M.R. Jr., and R.G. Northcutt (1983) Organization of the diencephalon and pretectum of the ray-finned fishes. In Fish Neurobiology, Vol. 2 (ed. by R.E. Davis and R.G. Northcutt), University of Michigan Press, Ann Arbor, pp. 117-163.

Butcher, L.L. (1983) Acetyicholinesterase histochemistry. In Methods in Chemical Neuroanatomy. Handbook for Chemical Neuroanatomy, Vol. 1 (ed. by A. Björklund and T. Hökfelt), Elsevier, Amsterdam, pp. 1-49.

Butler, A.B., M.F. Wullimann, and R.G. Northcutt (1989) Phylogeny of some pretectal visual nuclei in teleosts. Soc. Neurosci. Abstr., 15: 32.

Contestabile, A., P. Migani, A. Poli, L. Vilani, R. Bissoli, and G. Cristini (1980) Patterns of neurotransmitter function in the optic tectum of teleosts. Adv. Physiol. Sci., 31: 75-94.

Ekström, P. (1987) Distribution of choline acetyl-transferase-immunoreactive neurons in the brain of a cyprinid teleost (Phoxinux phoxinus L.). J. Comp. Neurol., 256: 494-515.

Fibiger, H.C. (1982) The organization and some projections of cholinergic neurons of the mammalian forebrain. Brain Res. Rev., 4: 327-388.

Godement, P., J. Vanselow, S. Thanos, and F. Bonhoeffer (1987) A study in developing visual systems with a new method of staining neurones and their processes in fixed tissue. Development, 101: 697-713.

Ito, H., and R. Kishida (1975) Organization of the teleostean nucleus rotundus. J. Morphol., 147: 89-107.

Koelle, G.B. (1950) The histochemical differentiation of types of cholinesterases and their localizations in tissues of the cat. $\mathbf{J}$. Pharmacol. Exp. Ther., 100: 158-179.

Koelle, G.B. (1951) The elimination of enzymatic diffusion artifacts in the histochemical localization of cholinesterases and a survey of their cellular distributions. J. Pharmacol. Exp. Ther., 103: 153-171.

Koelle, G.B. (1963) Cytological distributions and physiological functions of cholinesterases. In Cholinesterases and Anticholinesterase Agents. Handbook of Experimental Pharmacology, Vol. 15 (ed. by G.B. Koelle), Springer, Berlin, pp. 187-298.

Lauder, G.V., and K.F. Liem (1983) The evolution and interrelationships of the actinopterygian fishes. Bull. Mus. Comp. Zool., 150: 95-197.

Lynch, G., and H. Killackey (1974) Neuroanatomical techniques for neurobehavioral research. In The Neuropsychology of Aggression (ed. by R.E. Whalen), Plenum, New York, pp. 99-123.

Murakami, T., Y. Morita, and H. Ito (1986) Cytoarchitecture and fiber connections of the superficial pretectum in a teleost, Navodon modestus. Brain Res., 373: 213-221.

Northcutt, R.G. (1983) Evolution of the optic tectum in ray-finned fishes. In Fish Neurobiology, Vol. 2 (ed. by R.E. Davis and R.G. Northcutt), University of Michigan Press, Ann Arbor, pp. 1-42. 
Northcutt, R.G., and M.R. Braford, Jr. (1984) Some efferent connections of the superficial pretectum in the goldfish. Brain Res., 296: 181-184.

Northcutt, R.G., and M.F. Wullimann (1988) The visual system in teleost fishes: morphological patterns and trends. In Sensory biology of Aquatic Animals (ed. by J. Atema, R.R. Fay, A.N. Popper and W.N. Tavolga), Springer, New York, pp. 515-552.

Oswald, R.E., and J.A. Freeman (1980) Optic nerve transmitters in lower vertebrate species. Life Sci., 27: 527-533.

Oswald, R.E., D.E. Schmidt, and J.A. Freeman (1979) Assessment of acetylcholine as an optic nerve neurotransmitter in Bufo marinus. Neuroscience, 4: 1129-1136.

Romeis, B. (1989) Mikroskopische Technik. Urban \& Schwarzenberg, München.

Sakamoto, N., and H. Ito (1982) Fiber connections of the corpus glomerulosum in a teleost, Navodon modestus. J. Comp. Neurol., 205: 291-298.

Schmidt, A., G. Roth, and M. Ernst (1989) Distribution of substance P-like, leucine-enkephalin-like, and bombesin-like immunoreactivity and acetylcholinesterase activity in the visual system of salamanders. J. Comp. Neurol., 288: 123-135.

Sorenson, E.M., D. Parkinson, J.L. Dahl, and V.A. Chiapinelli (1989) Immunohistochemical localization of choline acetyltransferase in the chicken mesencephalon. J. Comp. Neurol., 281: 641-657.

Striedter, G.F., and R.G. Northcutt (1986) Connections of the superficial pretectum and nucleus isthmi in the sunfish Lepomis cyanellus. Soc. Neurosci. Abstr., 12: 103.

Striedter, G.F., and R.G. Northcutt (1989) Two distinct visual pathways through the superficial pretectum in a percomorph teleost. J. Comp. Neurol., 283: 342-354.
Wächtler, K. (1988) Phylogeny of the cholinergic synapse. In The Cholinergic Synapse (ed. by V.P. Whittaker), Springer, New York, pp. 57-80.

Wullimann, M.F. (1985) Zum Gehirn von Rhinecanthus aculeatus (Linnaeus 1758) (Balistidae, Tetraodontiformes, Teleostei). Inauguraldissertation Basel.

Wullimann, M.F. (1988) The tertiary gustatory center in sunfishes is not nucleus glomerulosus. Neurosci. Lett., 86: 6-10.

Wullimann, M.F., and D.L. Meyer (1989) Evolution of putative cholinergic visual pathways to the hypothalamus in teleost fishes. In Dynamics and Plasticity in Neuronal Systems (ed. by N. Elsner and W. Singer), Thieme, Stuttgart, p. 330.

Wullimann, M.F., and R.G. Northcutt (1986) Vision on the coral reef: some visual circuits in humu humus (Balistidae). Soc. Neurosci. Abstr., 12: 103.

Wullimann, M.F., and R.G. Northcutt (1989) Non-percomorph teleosts possess a homologue of nucleus glomerulosus. Soc. Neurosci. Abstr., 15: 32.

Zottoli, S.J., K.J. Rhodes, and E.J. Mufson (1987) Comparison of acetylcholinesterase and choline acetyltransferase staining patterns in the optic tectum of the goldfish Carassius auratus: a histochemical and immunocytochemical analysis. Brain Behav. Evol., 30: 143-159.

Dr. Mario F. Wullimann Abteilung Neuroanatomie Zentrum Anatomie Georg-August-Universität Kreuzbergring 36 D-3400 Göttingen (FRG) 